\title{
Acute mesenteric ischemia in a patient with severe cardiac disease
}

\author{
Camacho F. $\ddagger$, Amaral T. $\ddagger$, Braga A. ${ }^{\ddagger}$, Farinha F. $\ddagger$ \\ ${ }^{\ddagger}$ Department of Anaesthesiology, Centro Hospitalar de São João, Porto, Portugal
}

\section{Background}

Acute coronary syndrome, coupled with severe mitral disease with a few hours of onset, is an anaesthetic challenge. Perioperative handling, therapeutic choice and adequate monitoring are fundamental to maintain hemodynamic stability. The following case report describes a successful anaesthetic approach in a patient with a poor prognosis.

\section{Case report}

- 65 years old female, ASA VE, admitted to the emergency OR for exploratory laparotomy for suspicion of mesenteric ischemia.

- Past history: type II Diabetes Mellitus, Hypertension and Obesity.

- Admitted to the Cardiology ICU in the past $48 \mathrm{~h}$ for acute myocardial infarction complicated with pulmonary oedema and pleural effusion. Transthoracic Ecocardiography: severely compromised left ventricular function $(E F=22 \%)$ and severe mitral insufficiency with tendinous cord rupture.

- An intra-aortic balloon pump (IABP) was placed to maintain hemodynamic stability and she was scheduled for coronary bypass surgery and mitral valve replacement the next day. She had 2 episodes of atrial fibrillation with fast ventricular response that continued despite treatment. The IABP was removed 1 hour before surgery and heparin was suspended.

- After standard monitoring and placement of an arterial line, heart rate control was accomplished with 3 boluses of labetalol $1 \mathrm{mg}$.

- Anaesthetic induction was accomplished with midazolam $1 \mathrm{mg}$, etomidate $0.2 \mathrm{mg} / \mathrm{kg}$, fentanyl $0.15 \mathrm{mg}$ and rocuronium $1 \mathrm{mg} / \mathrm{kg}$.

- A central venous catheter was placed and 2 infusions were started: noradrenaline at $0.1 \mathrm{ug} / \mathrm{kg} / \mathrm{min}$ to maintain adequate blood pressure and isosorbide dinitrate because of the coronary disease.

- Anaesthetic maintenance with sevoflurane, fentanyl and morphine.

- The patient remained hemodynamically stable with no surgical or anaesthetic events.

- Diagnosis was confirmed and a bowel resection and colostomy were made. She was transferred to the ICU under assisted ventilation and sedated with midazolam and morphine.

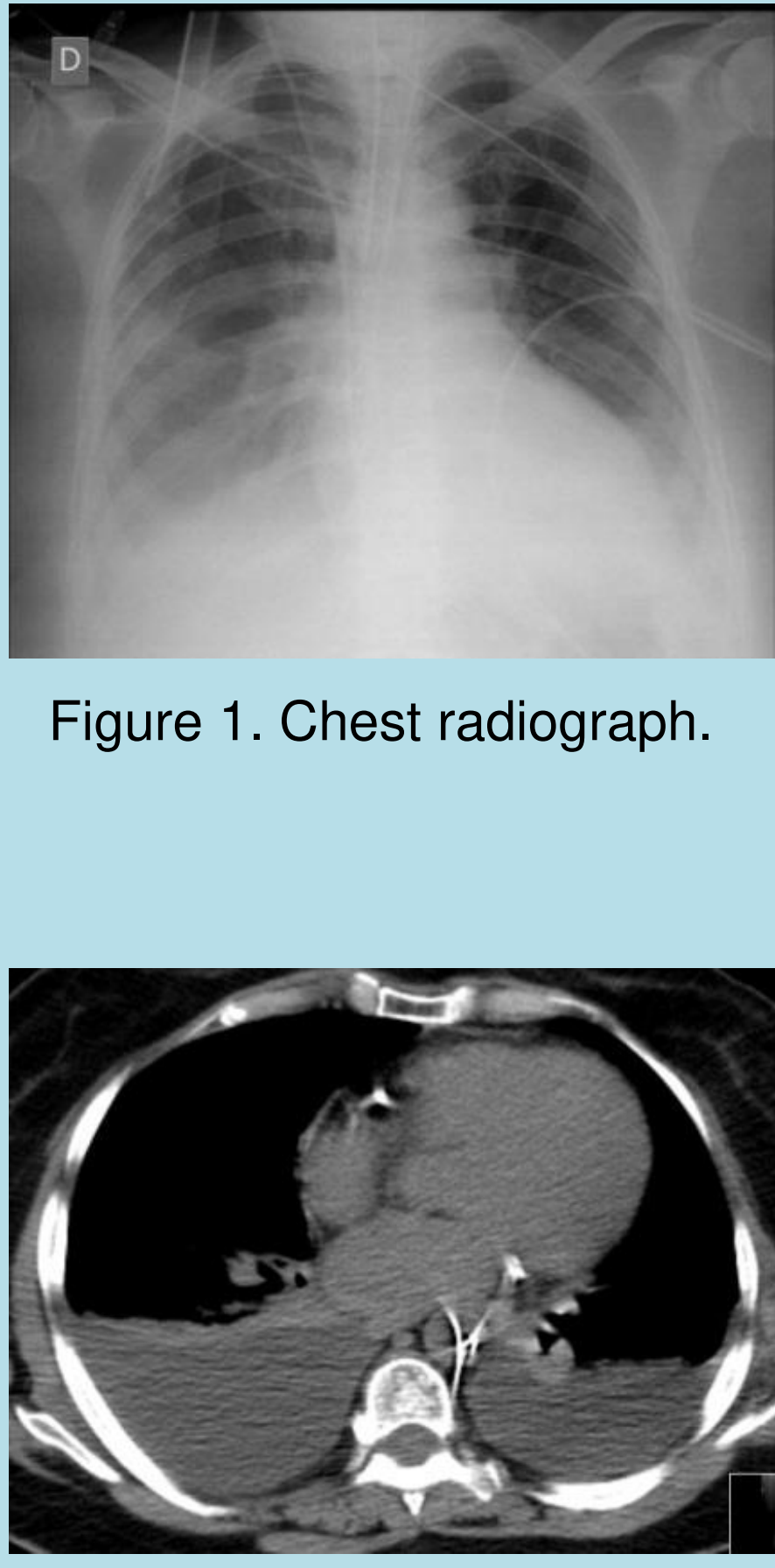

Figure 2. CT scan of the thorax.

\section{Discussion}

The serious cardiac and surgical disease of this patient predicted a poor prognosis. Difficulties maintaining hemodynamic stability were expected after anaesthetic induction and during the surgery. The choice of the anaesthetic approach and drugs is not an easy one and this case report shows a successful handling of a critical situation.

\section{Conclusion}

Patients with severe cardiac conditions submitted to urgent procedures require a careful anaesthetic management aiming for hemodynamic stability and outcome improvement.

\section{References}

